\title{
On Hamiltonian operators in differential algebras
}

\author{
Zharinov V.V. ${ }^{* \dagger}$
}

\begin{abstract}
Before we proposed an algebraic technics for the Hamiltonian approach to the evolution systems of partial differential equations, including systems with constraints. Here we further develop this approach and present the defining system of equations (suitable for the computer calculations), characterizing the Hamiltonian operators of the given form. We illustrate our technics by a simple example.
\end{abstract}

Keywords: differential algebra, Lie-Poisson structure, Jacobi identity, Hamiltonian operator, Hamiltonian evolution system.

\section{Introduction.}

In the previous paper [1] we proposed an algebraic technics (based on the book 2], Chapter VII) for the Hamiltonian approach to the evolution systems of partial differential equations, including systems with constraints. Here we further develop this approach and present the defining system of equations (suitable for the computer calculations) for the classification of the Hamiltonian operators of the given form.

We work in the frames of the algebra-geometrical approach to partial differential equations (see, for example, [2], 3], 4]). We hope that our results will be helpful in the studies connected with the papers [5] - [19].

We use the following general notations:

\footnotetext{
${ }^{*}$ Steklov Mathematical Institute
}

†E-mail: zharinov@mi.ras.ru 
- $\mathbb{F}=\mathbb{R}, \mathbb{C}, \quad \mathbb{N}=\{1,2,3, \ldots\} \subset \mathbb{Z}_{+}=\{0,1,2, \ldots\} ;$

- $\mathrm{M}=\{1, \ldots, m\}, \quad m \in \mathbb{N}$;

- $\mathbb{I}=\mathbb{Z}_{+}^{\mathrm{M}}=\left\{i=\left(i^{1}, \ldots, i^{m}\right) \mid i^{\mu} \in \mathbb{Z}_{+}, \mu \in \mathrm{M}\right\}$.

All linear operations are done over the number field $\mathbb{F}$. The summation over repeated upper and lower indices is as a rule assumed.

\section{The differential algebra.}

The main object in our construction is a differential algebra $\left(\mathcal{F}, \mathfrak{D}_{H}\right)$, where (see [1, for details and motivations):

- $\mathcal{F}$ is an unital (i.e., containing the unit element) associative commutative algebra;

- $\mathfrak{D}=\mathfrak{D}(\mathcal{F})$ is the Lie algebra of all differentiations of the algebra $\mathcal{F}$, it is assumed that $\mathfrak{D}=\mathfrak{D}_{V} \oplus_{\mathcal{F}} \mathfrak{D}_{H}$;

- $\mathfrak{D}_{V}=\mathfrak{D}_{V}(\mathcal{F})$ is the vertical subalgebra of the Lie algebra $\mathfrak{D}$ with the formal $\mathcal{F}$-basis $\partial=\left\{\partial_{a} \mid a \in \mathbf{A}\right\},\left[\partial_{a}, \partial_{b}\right]=0, a, b \in \mathbf{A}, \mathbf{A}$ is an index set, in particular, $\mathfrak{D}_{V}=\left\{X=X^{a} \partial_{a} \mid X^{a} \in \mathcal{F}\right\}$, where for any $L \in \mathcal{F}$ the action $\partial_{a} L \neq 0$ only for a finite number of indices $a \in \mathbf{A}$;

- $\mathfrak{D}_{H}=\mathfrak{D}_{H}(\mathcal{F})$ is the horizontal subalgebra of the Lie algebra $\mathfrak{D}$ with the $\mathcal{F}$-basis $D=\left\{D_{\mu} \mid \mu \in \mathrm{M}\right\},\left[D_{\mu}, D_{\nu}\right]=0, \mu, \nu \in \mathrm{M}$;

- $\left[D, \mathfrak{D}_{V}\right] \subset \mathfrak{D}_{V}$, i.e. $\left[D_{\mu}, V\right] \in \mathfrak{D}_{V}$ for all $\mu \in \mathrm{M}$ and $V \in \mathfrak{D}_{V}$.

Remind, that the set $\mathfrak{D}$ has two algebraic structures: the structure of a Lie algebra with the commutator $[X, Y]=X \circ Y-Y \circ X$, as the Lie bracket, and the structure of a $\mathcal{F}$-module with $(K \cdot X) L=K \cdot(X L)$, which are related by the matching condition $[X, L \cdot Y]=(X L) \cdot Y+L \cdot[X, Y]$ for all $K, L \in \mathcal{F}$, and for all $X, Y \in \mathfrak{D}$.

For any index sets $\mathbf{A}, \mathbf{B}$ the set $\mathcal{F}_{\mathbf{B}}^{\mathbf{A}}=\left\{\boldsymbol{\eta}=\left(\eta_{b}^{a}\right) \mid \eta_{b}^{a} \in \mathcal{F}, a \in \mathbf{A}, b \in \mathbf{B}\right\}$ has the structure of a $\mathcal{F}$-module with the component-wise multiplication, i.e., $K \cdot \boldsymbol{\eta}=\left(K \cdot \eta_{b}^{a}\right) \in \mathcal{F}_{\mathbf{B}}^{\mathbf{A}}$ for all $K \in \mathcal{F}, \boldsymbol{\eta} \in \mathcal{F}_{\mathbf{B}}^{\mathbf{A}}$.

We denote by $\mathcal{F}_{\mathbf{B}}^{\mathbf{A}}$ the $\mathcal{F}$-module of all elements $\boldsymbol{\eta}=\left(\eta_{b}^{a}\right) \in \mathcal{F}_{\mathbf{B}}^{\mathbf{A}}$, s.t. for any upper index $a \in \mathbf{A}$ only a finite number of components $\eta_{b}^{a} \neq 0$. In 
particular, $\stackrel{\circ}{\mathcal{F}}^{\mathbf{A}}=\mathcal{F}^{\mathbf{A}}$ for any index set $\mathbf{A}$, while $\stackrel{\circ}{\mathcal{F}}_{\mathbf{B}}$ consists of all finite elements $\boldsymbol{\eta}=\left(\eta_{b}\right) \in \mathcal{F}_{\mathbf{B}}$, and $\stackrel{\circ}{\mathcal{F}}_{\mathbf{B}}=\mathcal{F}_{\mathbf{B}}$ iff (i.e., if and only if) the index set $\mathbf{B}$ is finite. For example, by the above assumption, $\partial K=\left(\partial_{a} K\right) \in \stackrel{\circ}{\mathcal{F}}_{\mathbf{A}}$ for any $K \in \mathcal{F}$, in particular, the linear mapping $\partial: \mathcal{F} \rightarrow \stackrel{\circ}{\mathcal{F}}_{\mathbf{A}}$ is defined.

For any $\mathcal{F}_{\text {-module }} \mathcal{F}_{\mathbf{B}}^{\mathbf{A}}$ and any differentiation $X \in \mathfrak{D}$ the linear mapping $X: \mathcal{F}_{\mathbf{B}}^{\mathbf{A}} \rightarrow \mathcal{F}_{\mathbf{B}}^{\mathbf{A}}$ is defined component-wise, $\boldsymbol{\eta}=\left(\eta_{b}^{a}\right) \mapsto X \boldsymbol{\eta}=\left(X \eta_{b}^{a}\right)$. In particular, the Leibniz rule takes the form: $X(K \cdot \boldsymbol{\eta})=(X K) \cdot \boldsymbol{\eta}+K \cdot(X \boldsymbol{\eta})$ for all $X \in \mathfrak{D}, K \in \mathcal{F}, \boldsymbol{\eta} \in \mathcal{F}_{\mathbf{B}}^{\mathbf{A}}$.

With this notations, the commutator $\left[D_{\mu}, \partial_{a}\right]=\Gamma_{\mu a}^{b} \cdot \partial_{b}$ for all $\mu \in \mathrm{M}$, $a \in \mathbf{A}$, and we assume that the symbol $\Gamma=\left(\Gamma_{\mu a}^{b}\right) \in \stackrel{\circ}{\mathcal{F}}_{\mathbf{M} \mathbf{A}}^{\mathbf{A}}$. Note, that here the set $\mathrm{M}$ is finite, while the set $\mathbf{A}$ is as a rule infinite, and in the accordance with the above definition of the $\mathcal{F}$-module $\stackrel{\circ}{\mathcal{F}}_{\text {MA }}^{\mathbf{A}}$, for any index $b \in \mathbf{A}$ only a finite number of coefficients $\Gamma_{\mu a}^{b} \neq 0$.

\section{Horizontal differential operators.}

We call a linear mapping $P(D): \mathcal{F} \rightarrow \mathcal{F}$ (i.e., $\left.P(D) \in \operatorname{End}_{\mathbb{F}}(\mathcal{F})\right)$ a horizontal differential operator, if $P(D)=P_{i} \cdot D^{i}$, where the indices $i=\left(i^{1}, \ldots, i^{m}\right) \in \mathbb{I}$, coefficients $P=\left(P_{i}\right) \in \stackrel{\circ}{\mathcal{F}}_{\mathbb{I}}$, differential monomials $D^{i}=\left(D_{1}\right)^{i^{1}} \circ \ldots \circ\left(D_{m}\right)^{i^{m}}$, so $P(D) L=P_{i} \cdot D^{i} L$ for all $L \in \mathcal{F}$. The set of all horizontal differential operators we denote by $\mathcal{F}[D]$. It is an unital associative algebra with the the multiplication defined by the composition rule. In the same way for any four index sets $\mathbf{A}, \mathbf{B}, \mathbf{A}^{\prime}, \mathbf{B}^{\prime}$ the $\mathcal{F}[D]$-module $\mathcal{F}_{\mathbf{B}^{\prime} \mathbf{A}}^{\mathbf{A}^{\prime} \mathbf{B}}[D]$ of matrix horizontal differential operators

$$
P(D): \stackrel{\circ}{\mathcal{F}} \mathbf{\mathbf { A }} \rightarrow \stackrel{\circ}{\mathcal{F}}_{\mathbf{B}^{\prime}}^{\mathbf{A}^{\prime}}, \quad \boldsymbol{\eta}=\left(\eta_{b}^{a}\right) \mapsto \boldsymbol{\chi}=\left(\chi_{b^{\prime}}^{a^{\prime}}\right), \quad \chi_{b^{\prime}}^{a^{\prime}}=P_{b^{\prime} a i}^{a^{\prime} b} \cdot D^{i} \eta_{b}^{a},
$$

is defined, where $P=\left(P_{b^{\prime} a i}^{a^{\prime} b}\right) \in \stackrel{\circ}{\mathcal{F}}_{\mathbf{B}^{\prime} \mathbf{A} \mathbb{I}}^{\mathbf{A}^{\prime} \mathbf{B}}, P(D)=\left(P_{b^{\prime} a i}^{a^{\prime} b} \cdot D^{i}\right)$.

For every pair of index sets $\mathbf{A}, \mathbf{B}$ there is defined the natural pairing

$$
\langle\cdot, \cdot\rangle: \stackrel{\circ}{\mathcal{F}}_{\mathbf{B}}^{\mathbf{A}} \times \stackrel{\circ}{\mathcal{F}}_{\mathbf{A}}^{\mathbf{B}} \rightarrow \mathcal{F}, \quad \boldsymbol{\eta}=\left(\eta_{b}^{a}\right), \boldsymbol{\zeta}=\left(\zeta_{a}^{b}\right) \mapsto\langle\boldsymbol{\eta}, \boldsymbol{\zeta}\rangle=\eta_{b}^{a} \cdot \zeta_{a}^{b} .
$$

For every horizontal differential operator $P(D): \stackrel{\circ}{\mathcal{F}} \mathbf{A} \rightarrow \stackrel{\circ}{\mathcal{F}}_{\mathbf{B}^{\prime}}^{A^{\prime}}$ there is defined the Lagrange dual operator

$$
\stackrel{*}{P}(D): \stackrel{\circ}{\mathcal{F}}_{\mathbf{A}^{\prime}}^{\mathbf{B}^{\prime}} \rightarrow \stackrel{\circ}{\mathcal{F}} \mathbf{\mathbf { A }}, \quad \boldsymbol{\zeta}=\left(\zeta_{a^{\prime}}^{b^{\prime}}\right) \mapsto \omega=\left(\omega_{a}^{b}\right), \quad \omega_{a}^{b}=(-D)^{i}\left(\zeta_{a^{\prime}}^{b^{\prime}} \cdot P_{b^{\prime} a i}^{a^{\prime} b}\right),
$$


where $\stackrel{*}{P}=\left(\stackrel{*}{P} \underset{a b^{\prime} i}{b a^{\prime}}\right) \in \stackrel{\circ}{\mathcal{F}} \mathbf{A B}_{\mathbf{A B}^{\prime} \mathbb{I}}^{\mathbf{A}^{\prime}}, \stackrel{*}{P}(D)=\left(\stackrel{*}{P} \underset{a b^{\prime} i}{b a^{\prime}} \cdot D^{i}\right)=\left((-D)^{i} \circ P_{b^{\prime} a i}^{a^{\prime} b}\right)$, and the Green's formula (the integration by parts)

$$
\langle\boldsymbol{\zeta}, P(D) \boldsymbol{\eta}\rangle-\langle\stackrel{*}{P}(D) \boldsymbol{\zeta}, \boldsymbol{\eta}\rangle=D_{\mu} \psi^{\mu} \quad \text { for all } \quad \boldsymbol{\zeta} \in \stackrel{\circ}{\mathcal{F}}_{\mathbf{A}^{\prime}}^{\mathbf{B}^{\prime}}, \boldsymbol{\eta} \in \stackrel{\circ}{\mathcal{F}}_{\mathbf{A}}^{\mathbf{B}},
$$

holds with some current $\psi=\left(\psi^{\mu}(\boldsymbol{\zeta}, P(D), \boldsymbol{\eta})\right) \in \mathcal{F}^{\mathrm{M}}$.

Assumption 1. We assume that the differential algebra $\left(\mathcal{F}, \mathfrak{D}_{H}\right)$ is horizontally exact, i.e. a horizontal differential operator $P(D)=P_{i} \cdot D^{i}=0$ (i.e., $P(D) K=0$ for all $K \in \mathcal{F})$ iff the coefficients $P_{i}=0$ for all $i \in \mathbb{I}$.

Note, that in this case, the same is also true for matrix operators.

\section{The main ingredients}

The main components, instruments and assumptions here are (see [1], for more detail):

- the horizontal differential operator

$$
D: \mathcal{F} \rightarrow \mathcal{F}_{\mathrm{M}}, \quad K \mapsto D K=\left(D_{\mu} K\right)
$$

- the horizontal differential operator

$$
\operatorname{Div}=-\stackrel{*}{D}: \mathcal{F}^{\mathrm{M}} \rightarrow \mathcal{F}, \quad \psi=\left(\psi^{\mu}\right) \mapsto \operatorname{Div} \psi=D_{\mu} \psi^{\mu}
$$

- the horizontal differential operator

$$
\nabla: \mathcal{F}^{\mathbf{A}} \rightarrow \mathcal{F}_{\mathrm{M}}^{\mathbf{A}}, \quad \boldsymbol{\phi}=\left(\phi^{a}\right) \mapsto \boldsymbol{\eta}=\left(\eta_{\mu}^{a}\right), \quad \eta_{\mu}^{a}=D_{\mu} \phi^{a}+\Gamma_{\mu b}^{a} \cdot \phi^{b} ;
$$

- the Lagrange dual operator

$$
\stackrel{*}{\nabla}: \stackrel{\circ}{\mathcal{F}}_{\mathbf{A}}^{\mathrm{M}} \rightarrow \stackrel{\circ}{\mathcal{F}}_{\mathbf{A}}, \quad \chi=\left(\chi_{a}^{\mu}\right) \mapsto \mathbf{f}=\left(f_{a}\right), \quad f_{a}=-D_{\mu} \chi_{a}^{\mu}+\Gamma_{\mu a}^{b} \chi_{b}^{\mu}
$$

- the vertical differential operator $\partial: \mathcal{F} \rightarrow \stackrel{\circ}{\mathcal{F}}_{\mathbf{A}}, K \mapsto \partial K=\left(\partial_{a} K\right)$.

The operator $\nabla$ is correctly defined due to assumption $\left.\Gamma=\left(\Gamma_{\mu a}^{b}\right) \in \stackrel{\circ}{\mathcal{F}}_{\text {MA }}^{\mathbf{A}}\right)$. 
Proposition 1. The compositions $\partial \circ \operatorname{Div}, \stackrel{*}{\nabla} \circ \partial: \mathcal{F}^{\mathrm{M}} \rightarrow \stackrel{\circ}{\mathcal{F}} \mathbf{A}$, and the equality $\partial \circ \operatorname{Div}+\stackrel{*}{\nabla} \circ \partial=0$ holds.

Proof. Indeed, for any $\psi=\left(\psi^{\mu}\right) \in \mathcal{F}^{\mathrm{M}}$ we have

$$
\left(\partial \circ D_{\mu}\right) \psi^{\mu}+(\stackrel{*}{\nabla} \circ \partial) \psi^{\mu}=\left(-\left[D_{\mu}, \partial_{a}\right]+\Gamma_{\mu a}^{b} \partial_{b}\right) \psi^{\mu}=0
$$

in the accordance with the definition of the symbol $\Gamma$.

We also need:

- the linear subspace $\mathcal{E}=\operatorname{Ker} \nabla$ of the linear space $\mathcal{F}^{\mathbf{A}}$;

- the set $\mathfrak{D}_{E}=\mathfrak{D}_{E}(\mathcal{F})=\left\{V \in \mathfrak{D}_{V} \mid\left[D_{\mu}, V\right]=0, \mu \in \mathrm{M}\right\}$ of all evolutionary differentiations of the algebra $\mathcal{F}$.

The set $\mathfrak{D}_{E}$ is a subalgebra of the Lie algebra $\mathfrak{D}_{V}$, because $\left[\mathfrak{D}_{E}, \mathfrak{D}_{E}\right] \subset \mathfrak{D}_{E}$ due to the Jacobi identity for commutators, but it is not a submodule of the $\mathcal{F}$-module $\mathfrak{D}_{V}$ (see, e.g., [3]).

Proposition 2. The mapping ev : $\mathcal{E} \rightarrow \mathfrak{D}_{E}, \boldsymbol{\phi}=\left(\phi^{a}\right) \mapsto \mathrm{ev}_{\boldsymbol{\phi}}=\phi^{a} \cdot \partial_{a}$, is an isomorphism of linear spaces. Moreover, the structure of the Lie algebra on $\mathfrak{D}_{E}$ defines the isomorphic structure on $\mathcal{E}$ by the rule:

$$
[\boldsymbol{\phi}, \boldsymbol{\psi}]=\boldsymbol{\xi}, \quad \boldsymbol{\phi}=\left(\phi^{a}\right), \boldsymbol{\psi}=\left(\psi^{a}\right), \boldsymbol{\xi}=\left(\xi^{a}\right), \quad \xi^{a}=\mathrm{ev}_{\boldsymbol{\phi}} \psi^{a}-\mathrm{ev}_{\boldsymbol{\psi}} \phi^{a}, \quad a \in \mathbf{A} .
$$

Proof. The proof is done by the direct test.

Proposition 3. For every horizontal differential operator $P(D)=\left(P_{i b}^{a} \cdot D^{i}\right)$ : $\mathcal{F}^{\mathbf{B}} \rightarrow \mathcal{F}^{\mathbf{A}}$, for its Lagrange dual $\stackrel{*}{P}(D)=\left((-D)^{i} \circ P_{i b}^{a}\right): \stackrel{\circ}{\mathcal{F}}_{\mathbf{A}} \rightarrow \stackrel{\circ}{\mathcal{F}}_{\mathbf{B}}$ and for any $\boldsymbol{\phi} \in \mathcal{E}$ the following statements hold:

- $\left[\mathrm{ev}_{\boldsymbol{\phi}}, P(D)\right]=\mathrm{ev}_{\boldsymbol{\phi}} P(D)=\left(\left(\operatorname{ev}_{\boldsymbol{\phi}} P_{i b}^{a}\right) \cdot D^{i}\right)$;

$$
\text { - }\left[\operatorname{ev}_{\boldsymbol{\phi}}, \stackrel{*}{P}(D)\right]=\operatorname{ev}_{\boldsymbol{\phi}} \stackrel{*}{P}(D)=\left((-D)^{i} \circ\left(\operatorname{ev}_{\boldsymbol{\phi}} P_{i b}^{a}\right)\right)=\left[\mathrm{ev}_{\boldsymbol{\phi}}, P(D)\right]^{*} \text {, }
$$

i.e., the evolutionary differentiation $\mathrm{ev}_{\phi}$ acts here coefficient-wise.

Proof. The proof is based on the characteristic property of the evolutionary differentiations. 
Assumption 2. We assume that there exist an index set A and a horizontal differential operator $\mathbf{J}=J(D): \mathcal{F}^{\mathrm{A}} \rightarrow \mathcal{F}^{\mathbf{A}}, \phi=\left(\phi^{\alpha}\right) \mapsto \boldsymbol{\phi}=\left(\phi^{a}\right)$, s.t.,

(a) the composition $\nabla \circ \mathbf{J}=0$, i.e., $\operatorname{Im} \mathbf{J} \subset \operatorname{Ker} \nabla$,

(b) the commutator $\left.\left[\mathrm{ev}_{\boldsymbol{\phi}}, \mathbf{J}\right)\right]=0$ for all $\boldsymbol{\phi} \in \mathcal{E}$.

In more detail, $\mathbf{J}=\left(J_{\alpha i}^{a} \cdot D^{i}\right), J=\left(J_{\alpha i}^{a}\right) \in \stackrel{\circ}{\mathcal{F}}_{\mathrm{AI}}^{\mathbf{A}}, \phi^{a}=J_{\alpha i}^{a} \cdot D^{i} \phi^{\alpha}$.

Proposition 4. Let the horizontal differential operator $\stackrel{*}{\mathbf{J}}: \stackrel{\circ}{\mathcal{F}} \mathbf{A} \rightarrow \stackrel{\circ}{\mathcal{F}}_{\mathrm{A}}$ be the Lagrange dual to $\mathbf{J}: \mathcal{F}^{\mathrm{A}} \rightarrow \mathcal{F}^{\mathbf{A}}$, then

(a*) the composition $\mathbf{J}^{*} \circ \stackrel{*}{\nabla}=0$,

$\left(b^{*}\right) \quad$ the commutator $\left[\operatorname{ev}_{\boldsymbol{\phi}}, \mathbf{J}^{*}\right]=0$ for all $\boldsymbol{\phi} \in \mathcal{E}$.

Proof. The proof is done by the direct test.

\section{The Lie-Poisson structures.}

We shall use the notation:

- $\mathfrak{F}=\mathcal{F} / \operatorname{Im} \operatorname{Div}=\left\{\int K=K+\operatorname{Div} \mathcal{F}^{\mathrm{M}} \mid K \in \mathcal{F}\right\}$;

- $\mathcal{E}=\mathcal{F}^{\mathrm{A}}, \mathcal{E}^{*}=\operatorname{Hom}_{\mathcal{F}}(\mathcal{E} ; \mathcal{F})=\stackrel{\circ}{\mathcal{F}}_{\mathrm{A}}$.

Here, $\mathfrak{F}$ is the linear space of all functionals over $\mathcal{F}, \mathcal{E}$ is a linear space, and $\mathcal{E}^{*}$ is its dual. The natural projection $\mathcal{F} \rightarrow \mathfrak{F}$ is defined by the rule: $K \mapsto \int K=K+\operatorname{Div} \mathcal{F}^{\mathrm{M}}$.

Assumption 3. We assume that the differential algebra $\left(\mathcal{F}, \mathfrak{D}_{H}\right)$ is of the $d u$ Bois-Reymond type, i.e., it has the following property: for a given $K \in \mathcal{F}$ the equality $\int K \cdot L=0$ is valid for all $L \in \mathcal{F}$ iff $K=0$.

By Propositions 11 and 4, $\partial: \operatorname{Im} \operatorname{Div} \rightarrow \operatorname{Im} \stackrel{*}{\nabla}$ and $\stackrel{*}{\mathbf{J}} \circ \stackrel{*}{\nabla}=0$, hence we have the sequence of linear spaces:

$$
\mathcal{F} / \operatorname{Im} \operatorname{Div} \stackrel{\partial}{\longrightarrow} \stackrel{\circ}{\mathcal{F}}_{\mathbf{A}} / \operatorname{Im} \stackrel{*}{\nabla} \stackrel{\text { J }}{\longrightarrow} \stackrel{\circ}{\mathcal{F}}_{\mathrm{A}}
$$

In particular, the linear mapping (the variational derivative) 
• $\delta=\stackrel{*}{\mathbf{J}} \circ \partial: \mathfrak{F} \rightarrow \mathcal{E}^{*}, \quad \int K \mapsto \delta K=\stackrel{*}{\mathbf{J}}(\partial K)$,

is defined.

Definition 1. The Lie-Poisson structure over the differential algebra $\left(\mathcal{F}, \mathfrak{D}_{H}\right)$ is a bilinear mapping (the Lie-Poisson bracket)

$$
\{\cdot, \cdot\}: \mathfrak{F} \times \mathfrak{F} \rightarrow \mathfrak{F}, \quad \int K, \int L \mapsto\left\{\int K, \int L\right\},
$$

with the properties:

- $\left\{\int K, \int L\right\}+\left\{\int L, \int K\right\}=0 ; \quad$ (skew-symmetry)

- $\mathbf{J I}\left(\int K, \int L, \int M\right)=\left\{\int K,\left\{\int L, \int M\right\}\right\}+$ c.p. $=0 ; \quad$ (Jacobi identity)

where the abbreviation "c.p." stands for the cyclic permutation of arguments $\int K, \int L, \int M \in \mathfrak{F}$. In this case, the pair $(\mathfrak{F},\{\cdot, \cdot\})$ is a Lie algebra.

Definition 2 . We define the bracket $\{\cdot, \cdot\}$ by the rule:

$$
\left\{\int K, \int L\right\}=\int\langle\delta K, \Lambda(D) \delta L\rangle \text { for all } \int K, \int L \in \mathfrak{F}
$$

where a horizontal differential operator

$$
\Lambda(D): \mathcal{E}^{*} \rightarrow \mathcal{E}, \quad f=\left(f_{\alpha}\right) \mapsto \phi=\left(\phi^{\alpha}\right), \quad \phi^{\alpha}=\Lambda_{i}^{\alpha \beta} \cdot D^{i} f_{\beta}, \quad \alpha \in \mathrm{A},
$$

the set of the coefficients $\Lambda=\left(\Lambda_{i}^{\alpha \beta}\right) \in \stackrel{\circ}{\mathcal{F}}_{\mathbb{I}}^{\mathrm{AA}}$. Below we always assume that the operator $\Lambda(D)$ is the Lagrange skew-adjoint, i.e., $\Lambda(D)+\stackrel{*}{\Lambda}(D)=0$.

In this case, the bracket $\{\cdot, \cdot\}$ is skew-symmetric, and the problem left is to find a possibly simple and effective test for the operator $\Lambda(D)$ to be Hamiltonian, i.e., to ensure the Jacobi identity to be valid.

In the paper [1] we have proved the following algebraic analog of the Theorem 7.8 from the book [2].

Lemma 1. The Jacoby identity has the following representation:

$$
\mathbf{J I}\left(\int K, \int L, \int M\right)=\int\left\langle\delta K,\left[\operatorname{ev}_{\boldsymbol{\phi}(L)}, \Lambda(D)\right] \delta M\right\rangle+\text { c.p. }=0,
$$

where $\boldsymbol{\phi}(L)=(\mathbf{J} \circ \Lambda(D) \circ \delta) L, \Lambda(D)=\left(\Lambda_{i}^{\alpha \beta} \cdot D^{i}\right)$ is a horizontal differential skew-adjoint operator. 
Here we refine this result to a form more convenient for actual calculations.

Definition 3. We shall say that the Jacobi identity holds in the strengthened form if

$$
\int\left\langle f,\left[\operatorname{ev}_{\boldsymbol{\phi}(g)}, \Lambda(D)\right] h\right\rangle+\text { c.p. }=0,
$$

for all $f, g, h \in \mathcal{E}^{*}$, where $\boldsymbol{\phi}(g)=(\mathbf{J} \circ \Lambda(D)) g$.

Lemma 2. In the conditions of the Lemma 1 the Jacoby identity in the strengthened form can be written as

$R_{k l}^{\alpha \beta \gamma} \cdot D^{k} g_{\beta} \cdot D^{l} h_{\gamma}+(-D)^{l}\left(R_{k l}^{\beta \gamma \alpha} \cdot g_{\beta} \cdot D^{k} h_{\gamma}\right)+(-D)^{k}\left(R_{k l}^{\gamma \alpha \beta} \cdot D^{l} g_{\beta} \cdot h_{\gamma}\right)=0$

for all $g, h \in \mathcal{E}^{*}, \alpha, \beta, \gamma \in \mathrm{A}, k, l \in \mathbb{I}$, where the coefficients

$$
R_{k l}^{\alpha \beta \gamma}=R_{k l}^{\alpha \beta \gamma}(\Lambda)=\left(\begin{array}{c}
i \\
r
\end{array}\right) J_{\epsilon i}^{a} \cdot D^{i-r} \Lambda_{k-r}^{\epsilon \beta} \cdot \partial_{a} \Lambda_{l}^{\alpha \gamma} .
$$

Proof. We need to refine the expression

$$
\int\left\langle f,\left[\operatorname{ev}_{\boldsymbol{\phi}(g)}, \Lambda(D)\right] h\right\rangle=\int f_{\alpha} \cdot\left[\operatorname{ev}_{\boldsymbol{\phi}(g)}, \Lambda_{l}^{\alpha \gamma} \cdot D^{l}\right] h_{\gamma},
$$

where $f, g, h \in \mathcal{E}^{*}$. Here, $\boldsymbol{\phi}(g)=(\mathbf{J} \circ \Lambda(D)) g$, i.e.,

$$
\begin{aligned}
\phi^{a}(g) & =J_{\epsilon i}^{a} \cdot D^{i}\left(\Lambda_{k}^{\epsilon \beta} \cdot D^{k} g_{\beta}\right)=J_{\epsilon i}^{a} \cdot\left(\begin{array}{c}
i \\
r
\end{array}\right) \cdot D^{i-r} \Lambda_{k}^{\epsilon \beta} \cdot D^{k+r} g_{\beta} \\
& =\left(\begin{array}{c}
i \\
r
\end{array}\right) \cdot J_{\epsilon i}^{a} \cdot D^{i-r} \Lambda_{k-r}^{\epsilon \beta} \cdot D^{k} g_{\beta} .
\end{aligned}
$$

Hence, by Proposition 3 ,

$$
\begin{aligned}
\int f_{\alpha} \cdot\left[\operatorname{ev}_{\phi(g)}, \Lambda_{l}^{\alpha \gamma} \cdot D^{l}\right] h_{\gamma} & =\int f_{\alpha} \cdot\left(\begin{array}{l}
i \\
r
\end{array}\right) \cdot J_{\epsilon i}^{a} \cdot D^{i-r} \Lambda_{k-r}^{\epsilon \beta} \cdot D^{k} g_{\beta} \cdot \partial_{a} \Lambda_{l}^{\alpha \gamma} \cdot D^{l} h_{\gamma} \\
& =\int R_{k l}^{\alpha \beta \gamma} \cdot f_{\alpha} \cdot D^{k} g_{\beta} \cdot D^{l} h_{\gamma} .
\end{aligned}
$$

Thus,

$$
\begin{aligned}
& \int\left\langle\delta K,\left[\operatorname{ev}_{\phi(L)}, \Lambda(D)\right] \delta M\right\rangle+\text { c.p. } \\
& =\int R_{k l}^{\alpha \beta \gamma} \cdot\left(f_{\alpha} \cdot D^{k} g_{\beta} \cdot D^{l} h_{\gamma}+g_{\alpha} \cdot D^{k} h_{\beta} \cdot D^{l} f_{\gamma}+h_{\alpha} \cdot D^{k} f_{\beta} \cdot D^{l} g_{\gamma}\right) \\
& =\int\left(R_{k l}^{\alpha \beta \gamma} \cdot f_{\alpha} \cdot D^{k} g_{\beta} \cdot D^{l} h_{\gamma}+R_{k l}^{\beta \gamma \alpha} \cdot D^{l} f_{\alpha} \cdot g_{\beta} \cdot D^{k} h_{\gamma}+R_{k l}^{\gamma \alpha \beta} \cdot D^{k} f_{\alpha} \cdot D^{l} g_{\beta} \cdot h_{\gamma}\right) \\
& =\int f_{\alpha} \cdot\left(R_{k l}^{\alpha \beta \gamma} \cdot D^{k} g_{\beta} \cdot D^{l} h_{\gamma}+(-D)^{l}\left(R_{k l}^{\beta \beta \gamma} \cdot g_{\beta} \cdot D^{k} h_{\gamma}\right)+(-D)^{k}\left(R_{k l}^{\gamma \alpha \beta} \cdot D^{l} g_{\beta} \cdot h_{\gamma}\right)\right),
\end{aligned}
$$

where we twice used the Green's formula of the integration by parts. Clear, the last representation and the assumed du Bois-Reymond property of the differential algebra $\left(\mathcal{F}, \mathfrak{D}_{H}\right)$ imply our claim. 
Theorem 1. In the conditions of the Lemma 圆 the Jacoby identity in the strengthened form is reduced to the system: $Q=Q(\Lambda)=\left(Q_{k l}^{\alpha \beta \gamma}\right)=0$, or in more detail,

$$
Q_{k l}^{\alpha \beta \gamma}=0 \quad \text { for all } \quad \alpha, \beta, \gamma \in \mathrm{A}, \quad k, l \in \mathbb{I},
$$

where

$$
\left.Q_{k l}^{\alpha \beta \gamma}=Q_{k l}^{\alpha \beta \gamma}(\Lambda)=R_{k l}^{\alpha \beta \gamma}+(-1)^{j}\left(_{p, k, l-i}^{j}\right) D^{p} R_{i j}^{\beta \gamma \alpha}+(-1)^{i}{ }_{p, k-j, l}^{i}\right) D^{p} R_{i j}^{\gamma \alpha \beta},
$$

and $\left(\begin{array}{c}j \\ p, k, l-i\end{array}\right),\left(\begin{array}{c}i \\ p, k-j, l\end{array}\right)$ are the trinomial coefficients 1 .

Proof. Indeed, here

$$
\begin{aligned}
& R_{k l}^{\alpha \beta \gamma} \cdot D^{k} g_{\beta} \cdot D^{l} h_{\gamma}+(-D)^{l}\left(R_{k l}^{\beta \gamma \alpha} \cdot g_{\beta} \cdot D^{k} h_{\gamma}\right)+(-D)^{k}\left(R_{k l}^{\gamma \alpha \beta} \cdot D^{l} g_{\beta} \cdot h_{\gamma}\right) \\
& =R_{k l}^{\alpha \beta \gamma} \cdot D^{k} g_{\beta} \cdot D^{l} h_{\gamma}+(-1)^{j}\left(\begin{array}{c}
j \\
p q r
\end{array}\right) D^{p} R_{k j}^{\beta \gamma \alpha} \cdot D^{q} g_{\beta} \cdot d^{r+k} h_{\gamma} \\
& +(-1)^{i}\left(\begin{array}{c}
i \\
p q r
\end{array}\right) D^{p} R_{i l}^{\gamma \alpha \beta} \cdot D^{q+l} g_{\beta} \cdot D^{r} h_{\gamma} \\
& =R_{k l}^{\alpha \beta \gamma} \cdot D^{k} g_{\beta} \cdot D^{l} h_{\gamma}+(-1)^{j}\left(\begin{array}{c}
j \\
p, k, l-i
\end{array}\right) D^{p} R_{i j}^{\beta \gamma \alpha} \cdot D^{k} g_{\beta} \cdot D^{l} h_{\gamma} \\
& +(-1)^{i}\left(\begin{array}{c}
i \\
p, k-j, l
\end{array}\right) D^{p} R_{i j}^{\gamma \alpha \beta} \cdot D^{k} g_{\beta} \cdot D^{l} h_{\gamma}=Q_{k l}^{\alpha \beta \gamma} \cdot D^{k} g_{\beta} \cdot D^{l} h_{\gamma} .
\end{aligned}
$$

To complete the proof it is enough to use the assumed horizontal exactness of the differential algebra $\left(\mathcal{F}, \mathfrak{D}_{H}\right)$.

\section{Evolution without constraints.}

Here (see 11, for more detail), the algebra $\mathcal{F}=\mathcal{C}_{\text {fin }}^{\infty}(\mathrm{XU})$ is the algebra of all smooth functions on the infinite dimensional space $\mathbf{X} \mathbf{U}=\mathrm{X} \times \mathbf{U}$, depending on a finite number of the arguments $x^{\mu}, u_{i}^{\alpha}$, where

- $\mathrm{X}=\mathbb{R}^{\mathrm{M}}=\left\{x=\left(x^{\mu}\right) \mid x^{\mu} \in \mathbb{R}, \mu \in \mathrm{M}\right\}$ is the space of independent variables;

- $\mathrm{U}=\mathbb{R}^{\mathrm{A}}=\left\{u=\left(u^{\alpha}\right) \mid u^{\alpha} \in \mathbb{R}, \alpha \in \mathrm{A}\right\}$ is the space of dependent variables, $\mathrm{A}$ is a finite index set;

- $\mathbf{U}=\mathbb{R}_{\mathbb{I}}^{\mathrm{A}}=\left\{\mathbf{u}=\left(u_{i}^{\alpha}\right) \mid u_{i}^{\alpha} \in \mathbb{R}, \alpha \in \mathrm{A}, i \in \mathbb{I}\right\}$ is the space of differential variables, $u^{\alpha}=u_{0}^{\alpha}$.

\footnotetext{
${ }^{1}$ Remind, the polynomial coefficients are $\left(\begin{array}{c}i \\ j_{1} \ldots j_{p}\end{array}\right)=\frac{i !}{j_{1} ! \ldots j_{p} !}, i=j_{1}+\ldots j_{p}$. In particular, the binomial coefficient $\left(\begin{array}{l}i \\ j\end{array}\right)=\left(\begin{array}{c}i \\ j, i-j\end{array}\right)$.
} 
The Lie subalgebra $\mathfrak{D}_{H}$ has the $\mathcal{F}$-basis $D=\left\{D_{\mu} \mid \mu \in \mathrm{M}\right\}$, where the total partial derivatives

$$
D_{\mu}=\partial_{x^{\mu}}+u_{i+(\mu)}^{\alpha} \partial_{u_{i}^{\alpha}}, \quad i+(\mu)=\left(i^{1}, \ldots, i^{\mu}+1, \ldots, i^{m}\right) .
$$

The Lie subalgebra $\mathfrak{D}_{V}$ has the $\mathcal{F}$-basis $\left\{\partial_{u_{i}^{\alpha}} \mid \alpha \in \mathrm{A}, i \in \mathbb{I}\right\}$, thus, here, $a=\left(\begin{array}{c}\alpha \\ i\end{array}\right), \mathbf{A}=\left(\begin{array}{l}\mathrm{A} \\ \mathbb{I}\end{array}\right)$, and the commutator

$$
\left[D_{\mu}, \partial_{u_{i}^{\alpha}}\right]=-\partial_{u_{i-(\mu)}^{\alpha}}, \quad \text { i.e., } \quad \Gamma_{\mu \alpha j}^{i \beta}=-\delta_{\alpha}^{\beta} \delta_{j+(\mu)}^{i}, \quad \alpha, \beta \in \mathrm{A}, i, j \in \mathbb{I}, \mu \in \mathrm{M} \text {. }
$$

Note, the pair $\left(\begin{array}{l}\beta \\ j\end{array}\right)$ is the single upper index, while $\left(\begin{array}{l}i \\ \alpha\end{array}\right)$ is the single lower index.

Further, in this situation,

- $\nabla: \mathcal{F}_{\mathbb{I}}^{\mathrm{A}} \rightarrow \mathcal{F}_{\mathrm{MI}}^{\mathrm{A}}, \quad \phi=\left(\phi_{i}^{\alpha}\right) \mapsto \boldsymbol{\eta}=\left(\eta_{\mu i}^{\alpha}\right), \quad \eta_{\mu i}^{\alpha}=D_{\mu} \phi_{i}^{\alpha}-\phi_{i+(\mu)}^{\alpha} ;$

- $\mathbf{j}: \mathcal{F}^{\mathrm{A}} \rightarrow \mathcal{F}_{\mathbb{I}}^{\mathrm{A}}, \quad \phi=\left(\phi^{\alpha}\right) \mapsto \boldsymbol{\phi}=\left(\phi_{i}^{\alpha}\right), \quad \phi_{i}^{\alpha}=D^{i} \phi^{\alpha}=\delta_{i k} \delta_{\beta}^{\alpha} \cdot D^{k} \phi^{\beta} ;$

- $\operatorname{Ker} \mathbf{j}=0, \quad \operatorname{Im} \mathbf{j}=\operatorname{Ker} \nabla, \quad\left[\mathrm{ev}_{\boldsymbol{\phi}}, \mathbf{j}\right]=0$ for all $\boldsymbol{\phi} \in \mathcal{E}$;

- $Q_{k l}^{\alpha \beta \gamma}=R_{k l}^{\alpha \beta \gamma}+(-1)^{j}\left(\begin{array}{c}j \\ p, k, l-i\end{array}\right) D^{p} R_{i j}^{\beta \gamma \alpha}+(-1)^{i}\left(\begin{array}{c}i \\ p, k-j, l\end{array}\right) D^{p} R_{i j}^{\gamma \alpha \beta}$;

- $R_{k l}^{\alpha \beta \gamma}=\left(\begin{array}{l}i \\ r\end{array}\right) D^{i-r} \Lambda_{k-r}^{\epsilon \beta} \cdot \partial_{u_{i}^{\epsilon}} \Lambda_{l}^{\alpha \gamma}, \quad \alpha, \beta, \gamma \in \mathrm{A}, \quad k, l, i, r, j, p \in \mathbb{I}$.

\section{The simplest case.}

Here,

- $\mathrm{X}=\mathbb{R}, \quad \mathrm{U}=\mathbb{R}, \quad \mathrm{U}=\mathbb{R}_{\mathbb{I}}, \quad \mathbb{I}=\mathbb{Z}_{+}, \quad \mathcal{F}=\mathcal{C}_{\text {fin }}^{\infty}(\mathrm{XU}) ;$

- $D=\partial_{x}+u_{i+1} \partial_{u_{i}}, \quad\left[D, \partial_{u_{i}}\right]=-\partial_{u_{i-1}}, \quad i \in \mathbb{I}$.

Further in this case, the horizontal differential operator $\Lambda(D)=\sum_{i=0}^{s} \Lambda_{i} \cdot D^{i}$, where the order $s \in \mathbb{N}$, the coefficients $\Lambda_{i}=\Lambda_{i}\left(u_{0}, u_{1}, \ldots, u_{n(i)}\right) \in \mathcal{F}, 0 \leq$ $i \leq s$. In particular (see Lemma 2, Theorem 1),

- $R_{k l}=\left(\begin{array}{c}i \\ r\end{array}\right) D^{i-r} \Lambda_{k-r} \cdot \partial_{u_{i}} \Lambda_{l}, \quad k, l, i, r, j, p \in \mathbb{I}$;

- $Q_{k l}=R_{k l}+(-1)^{j}\left(\begin{array}{c}j \\ p, k, l-i\end{array}\right) D^{p} R_{i j}+(-1)^{i}\left(\begin{array}{c}i \\ p, k-j, l\end{array}\right) D^{p} R_{i j}$;

- $\operatorname{supp} R=\left\{(k, l) \in \mathbb{I}^{2} \mid l \leq s, \quad k \leq n(l)+s\right\}$; 
- sp $R_{k l}=\left\{(r, i) \in \mathbb{I}^{2} \mid \max \{0, k-s\} \leq r \leq k, r \leq i \leq n(l)\right\}$.

Proposition 5. With these notations,

$$
R_{k l}=\sum_{s p R_{k l}}\left(\begin{array}{c}
i \\
r
\end{array}\right) D^{i-r} \Lambda_{k-r} \cdot \partial_{u_{i}} \Lambda_{l}, \quad(k, l) \in \operatorname{supp} R,
$$

and $R_{k l}=0$ for $(k, l) \notin \operatorname{supp} R$.

Further, we split

$$
Q_{k l}=R_{k l}+Q_{k l}^{\prime}+Q_{k l}^{\prime \prime}, \quad(k, l) \in \operatorname{supp} Q,
$$

where $\operatorname{supp} Q=\operatorname{supp} R \cup \operatorname{supp} Q^{\prime} \cup \operatorname{supp} Q^{\prime \prime} \subset \mathbb{I}^{2}$,

- $\operatorname{supp} Q^{\prime}=\cup_{(i, j) \in \operatorname{supp} R}\left\{(k, l) \in \mathbb{I}^{2} \mid k \leq j, l \geq i, k+l \leq i+j\right\}$,

- $\operatorname{supp} Q^{\prime \prime}=\cup_{(i, j) \in \operatorname{supp} R}\left\{(k, l) \in \mathbb{I}^{2} \mid k \geq j, l \leq i, k+l \leq i+j\right\}$.

By definition, $R_{k l}=0$ for $(k, l) \notin \operatorname{supp} R, Q_{k l}^{\sharp}=0$ for $(k, l) \notin \operatorname{supp} Q^{\sharp}$, where $\sharp=^{\prime}, "$.

\subsection{The example.}

Let us consider the operator $\Lambda(D)=\lambda D+\frac{1}{2} D \lambda, \lambda=\lambda\left(u_{0}, \ldots, u_{n}\right) \in \mathcal{F}$, $n \in \mathrm{Z}_{+}$. Here, $s=1, \Lambda_{0}=\frac{1}{2} D \lambda, \Lambda_{1}=\lambda, n(i)=n+1-i$ for $i=0,1$, hence, $n(i)+i=n+1$. The operator $\Lambda(D)$ is the Lagrange skew-adjoint by construction. According to Theorem 1 1 , the function $\lambda$ is defined by the system of differential equations $Q_{k l}=Q_{k l}(\lambda)=0, k, l \in \mathbb{I}$.

As one can calculate, $\operatorname{supp} R=\left\{(k, l) \in \mathbb{I}^{2} \mid l \leq 1, k \leq n(l)+1\right\}$;

Let us denote $\lambda_{u_{i}}=\partial_{u_{i}} \lambda, i=0, \ldots n$, and set

$$
\left(\begin{array}{l}
i \\
k
\end{array}\right)^{\prime}=\left(\begin{array}{c}
i \\
k
\end{array}\right)+2\left(\begin{array}{c}
i \\
k-1
\end{array}\right), \quad\left(\begin{array}{c}
i \\
p k l
\end{array}\right)^{\prime}=\left(\begin{array}{c}
i \\
p, k-1, l
\end{array}\right)-\left(\begin{array}{c}
i \\
p, k, l-1
\end{array}\right)=\frac{(k-l) i !}{p ! k ! l !}, p+k+l=i+1 .
$$

Now (remind, we assume the summation over the repeated upper and lower indices in the natural limits),

$$
\begin{aligned}
R_{k l} & =\left(\begin{array}{c}
i \\
r
\end{array}\right) D^{i-r} \Lambda_{k-r} \cdot \partial_{u_{i}} \Lambda_{l}=(k-r=0,1)= \\
& =\left(\left(\begin{array}{c}
i \\
k
\end{array}\right) D^{i-k} \Lambda_{0}+\left(\begin{array}{c}
i \\
k-1
\end{array}\right) D^{i+1-k} \Lambda_{1}\right) \cdot \partial_{u_{i}} \Lambda_{l}= \\
& \left.=\frac{1}{2}\left(\begin{array}{c}
i \\
k
\end{array}\right) D^{i+1-k} \lambda+2\left(\begin{array}{c}
i \\
k-1
\end{array}\right) D^{i+1-k} \lambda\right) \cdot \partial_{u_{i}} \Lambda_{l}=\frac{1}{2}\left(\begin{array}{c}
i \\
k
\end{array}\right)^{\prime} D^{i+1-k} \lambda \cdot \partial_{u_{i}} \Lambda_{l} .
\end{aligned}
$$


In particular,

$$
R_{k 1}=\frac{1}{2}\left(\begin{array}{c}
i \\
k
\end{array}\right)^{\prime} D^{i+1-k} \lambda \cdot \lambda_{u_{i}}, \quad k=0, \ldots, n+1,
$$

while

$$
\begin{aligned}
R_{k 0} & =\frac{1}{4}\left(\begin{array}{l}
i \\
k
\end{array}\right)^{\prime} D^{i+1-k} \lambda \cdot \partial_{u_{i}}(D \lambda)=\left(\partial_{u_{i}} \circ D=D \circ \partial_{u_{i}}+\partial_{u_{i-1}}\right)= \\
& =\frac{1}{4}\left(\begin{array}{c}
i \\
k
\end{array}\right)^{\prime} D^{i+1-k} \lambda \cdot D \lambda_{u_{i}}+\left(\begin{array}{c}
i \\
k
\end{array}\right)^{\prime} D^{i+1-k} \lambda \cdot \lambda_{u_{i-1}}=\left(\left(\begin{array}{c}
i+1 \\
k
\end{array}\right)^{\prime}-\left(\begin{array}{c}
i \\
k
\end{array}\right)^{\prime}=\left(\begin{array}{c}
i \\
k-1
\end{array}\right)^{\prime}\right)= \\
& =\frac{1}{4}\left(\left(\begin{array}{c}
i \\
k
\end{array}\right)^{\prime}\left(D^{i+2-k} \lambda \cdot \lambda_{u_{i}}+D^{i+1-k} \lambda \cdot D \lambda_{u_{i}}\right)+\left(\begin{array}{c}
i \\
k-1
\end{array}\right)^{\prime} D^{i+2-k)} \lambda \cdot \lambda_{u_{i}}\right)= \\
& =\frac{1}{4}\left(D\left(\left(\begin{array}{c}
i \\
k
\end{array}\right)^{\prime} D^{i+1-k} \lambda \cdot \lambda_{u_{i}}\right)+\left(\begin{array}{c}
i \\
k-1
\end{array}\right)^{\prime} D^{i+1-(k-1)} \lambda \cdot \lambda_{u_{i}}\right),
\end{aligned}
$$

that is

$$
R_{k 0}=\frac{1}{2}\left(D R_{k 1}+R_{k-1,1}\right), \quad k=0, \ldots, n+2 .
$$

Further,

$$
\begin{aligned}
Q_{k l}^{\prime} & =(-1)^{j}\left(\begin{array}{c}
j \\
p, k, l-i
\end{array}\right) D^{p} R_{i j}=(j=0,1)=\left(\begin{array}{c}
0 \\
p, k, l-i
\end{array}\right) D^{p} R_{i 0}-\left(\begin{array}{c}
1 \\
p, k, l-i
\end{array}\right) D^{p} R_{i 1}= \\
& =\delta_{k}^{0} R_{l 0}-\delta_{k}^{0} D R_{l 1}-\delta_{k+(l-i)}^{1} R_{i 1}=\left(D R_{l 1}+R_{l-1,1}=2 R_{l 0}\right)= \\
& =-\left(\delta_{k}^{0} R_{l 0}+\delta_{k}^{1} R_{l 1}\right)=-R_{l k},
\end{aligned}
$$

that is

$$
Q_{k l}^{\prime}=-R_{l k}, \quad l=0, \ldots, n+2, \quad k=0,1
$$

Still further,

$$
\begin{aligned}
Q_{k l}^{\prime \prime} & =(-1)^{i}\left(\begin{array}{c}
i \\
p, k-j, l
\end{array}\right) D^{p} R_{i j}=(j=0,1)= \\
& =(-1)^{i}\left(\begin{array}{c}
i \\
p k l
\end{array}\right) D^{p} R_{i 0}+(-1)^{i}\left(\begin{array}{c}
i \\
p, k-1, l
\end{array}\right) D^{p} R_{i 1}=\left(2 R_{i 0}=D R_{i 1}+R_{i-1,1}\right)= \\
& =\frac{1}{2}(-1)^{i}\left(\begin{array}{c}
i \\
p k l
\end{array}\right) D^{p}\left(D R_{i 1}+R_{i-1,1}\right)+(-1)^{i}\left(\begin{array}{c}
i \\
p, k-1, l
\end{array}\right) D^{p} R_{i 1}= \\
& \left.=\frac{1}{2}(-1)^{i}\left(\begin{array}{c}
i \\
p-1, k, l
\end{array}\right)-\left(\begin{array}{c}
i+1 \\
p k l
\end{array}\right)+2\left(\begin{array}{c}
i \\
p, k-1, l
\end{array}\right)\right) D^{p} R_{i 1}= \\
& \left.=\frac{1}{2}(-1)^{i}\left(\begin{array}{c}
i \\
p, k-1, l
\end{array}\right)-\left(\begin{array}{c}
i \\
p, k, l-1
\end{array}\right)\right) D^{p} R_{i 1},
\end{aligned}
$$

where we used the equality

$$
\left(\begin{array}{c}
i \\
p-1, k, l
\end{array}\right)+\left(\begin{array}{c}
i \\
p, k-1, l
\end{array}\right)+\left(\begin{array}{c}
i \\
p, k, l-1
\end{array}\right)=\left(\begin{array}{c}
i+1 \\
p k l
\end{array}\right), \quad \text { remind } \quad p+k+l=i+1 .
$$

Thus,

$$
Q_{k l}^{\prime \prime}=\frac{(-1)^{i}}{2}\left(\begin{array}{c}
i \\
p k l
\end{array}\right)^{\prime} D^{p} R_{i 1}, \quad i=0, \ldots, n+1, \quad p+k+l=i+1 \leq n+2 .
$$


Note, $Q_{k l}^{\prime \prime}=-Q_{l k}^{\prime \prime}$ due to the equality $\left(\begin{array}{c}i \\ p k l\end{array}\right)^{\prime}=-\left(\begin{array}{c}i \\ p l k\end{array}\right)^{\prime}$ (see the definition).

At last,

$$
Q_{k l}=Q_{k l}(\lambda)=R_{k l}-R_{l k}+\frac{(-1)^{i}}{2}\left(\begin{array}{c}
i \\
p k l
\end{array}\right)^{\prime} D^{p} R_{i 1}=-Q_{l k} .
$$

Our aim now is to find all functions $\lambda=\lambda\left(u_{0}, \ldots, u_{n}\right) \in \mathcal{F}, n \in \mathbb{Z}_{+}$, such that $Q(\lambda)=\left(Q_{k l}(\lambda)\right)=0$. It is convenient to start with the equation $Q_{1, n+1}=0, n \in \mathbb{N}$. Here, $R_{1, n+1}=0, R_{n+1,1}=\lambda \cdot \lambda_{u_{n}}$, while

$$
Q_{1, n+1}^{\prime \prime}=\frac{(-1)^{i}}{2}\left(\begin{array}{c}
i \\
p, 1, n+1
\end{array}\right)^{\prime} D^{p} R_{i 1}=\frac{(-1)^{n+1}}{2}\left(\begin{array}{c}
n+1 \\
0,1, n+1
\end{array}\right)^{\prime} R_{n+1,1}=\frac{(-1)^{n} n}{2} R_{n+1,1} .
$$

Thus,

$$
Q_{1, n+1}=-R_{n+1,1}+\frac{(-1)^{n} n}{2} R_{n+1,1}=\left(\frac{(-1)^{n} n}{2}-1\right) R_{n+1,1}\left\{\begin{array}{l}
=0, n=2 \\
\neq 0, n>2
\end{array} .\right.
$$

Hence, the equation $Q_{1, n+1}=0$ implies $\partial_{u_{n}} \lambda=0$ for $n>2$, in other words, $\lambda=\lambda\left(u_{0}, u_{1}, u_{2}\right)$. The case $n=2$ needs further detailed research. Namely, here,

$$
\begin{aligned}
& Q_{04}=0-R_{40}+\frac{(-1)^{2}}{2} R_{31}=-\frac{1}{2} R_{31}+\frac{(-1)^{2}}{2} R_{31}=0 \\
& Q_{03}=0-R_{30}-\frac{1}{2} R_{21}+\frac{3}{2} D R_{31}=D R_{31}-R_{21} \\
& Q_{02}=0-R_{20}+\frac{1}{2}\left(3 D^{2} R_{31}-2 D R_{21}+R_{11}\right)=\frac{3}{2} D\left(D R_{31}-R_{21}\right)=\frac{3}{2} D Q_{03} \\
& Q_{01}=R_{01}-R_{10}+\frac{1}{2}\left(D^{3} R_{31}-D^{2} R_{21}+D R_{11}-R_{01}\right)=\frac{1}{2} D^{2} Q_{03} \\
& Q_{13}=0-R_{31}+R_{31}=0 \\
& Q_{12}=0-R_{21}+\left(\frac{3}{2} D R_{31}-\frac{1}{2} R_{21}\right)=\frac{3}{2} Q_{03}
\end{aligned}
$$

where we used the formulas (2) and (5). All other equations are non relevant. Thus, the only relevant equation is the following: $Q_{03}=D R_{31}-R_{21}=0$. Here,

$R_{31}=\lambda \cdot \lambda_{u_{2}}, \quad D R_{31}=D \lambda \cdot \lambda_{u_{2}}+\lambda \cdot D \lambda_{u_{2}}, \quad R_{21}=\lambda \cdot \lambda_{u_{1}}+\frac{5}{2} D \lambda \cdot \lambda_{u_{2}}$, $Q_{03}=\lambda \cdot D \lambda_{u_{2}}-\frac{3}{2} D \lambda \cdot \lambda_{u_{2}}-\lambda \cdot \lambda_{u_{1}}=u_{3}\left(\lambda \cdot \lambda_{u_{2} u_{2}}-\frac{3}{2} \lambda_{u_{2}}^{2}\right)+\tilde{Q}_{03}\left(u_{0}, u_{1}, u_{2}\right)$.

The equation $\lambda \cdot \lambda_{u_{2} u_{2}}-\frac{3}{2} \lambda_{u_{2}}^{2}=0$ has the general solution $\lambda=\left(\phi u_{2}+\psi\right)^{-2}$, where the functions $\phi, \psi\left(u_{0}, u_{1}\right) \in \mathcal{F}$ are arbitrary. The reduced equation has the form

$$
\tilde{Q}_{03}=\lambda \cdot\left(\lambda_{u_{1} u_{2}} u_{2}+\lambda_{u_{0} u_{2}} u_{1}\right)-\frac{3}{2}\left(\lambda_{u_{1}} u_{2}+\lambda_{u_{0}} u_{1}\right) \cdot \lambda_{u_{2}}-\lambda \cdot \lambda_{u_{1}}=0
$$


where

$$
\begin{aligned}
& \lambda_{u_{i}}=-2\left(\phi u_{2}+\psi\right)^{-3}\left(\phi_{u_{i}} u_{2}+\psi_{u_{i}}\right), i=0,1, \quad \lambda_{u_{2}}=-2\left(\phi u_{2}+\psi\right)^{-3} \phi, \\
& \lambda_{u_{i} u_{2}}=2\left(\phi u_{2}+\psi\right)^{-4}\left(3\left(\phi_{u_{i}} u_{2}+\psi_{u_{i}}\right) \phi-\left(\phi u_{2}+\psi\right) \phi_{u_{i}}\right), i=0,1 .
\end{aligned}
$$

After the substitution and some simple computations we get the final form of this equation

$$
\tilde{Q}_{03}=2\left(\phi u_{2}+\psi\right)^{-5}\left(\psi_{u_{1}}-\phi_{u_{0}} u_{1}\right)=0
$$

The resulting equation $\psi_{u_{1}}-\phi_{u_{0}} u_{1}=0$ has the general solution

$$
\phi\left(u_{0}, u_{1}\right)=\left.\chi_{v}(u, v)\right|_{u=u_{0}, v=u_{1}^{2} / 2}, \quad \psi\left(u_{0}, u_{1}\right)=\left.\chi_{u}(u, v)\right|_{u=u_{0}, v=u_{1}^{2} / 2},
$$

where $\chi(u, v) \in \mathcal{C}^{\infty}\left(\mathbb{R}^{2}\right)$ is an arbitrary smooth function.

We summarize our calculations as the following theorem.

Theorem 2 (cf. [7], [8]). The differential operator $\Lambda(D)=\lambda D+\frac{1}{2} D \lambda, \lambda \in \mathcal{F}$, is Hamiltonian iff $\lambda=\left(\phi u_{2}+\psi\right)^{-2}$, where the functions $\phi, \psi\left(u_{0}, u_{1}\right) \in \mathcal{F}$ are defined by the formulas (6).

Acknowledgments. This work is supported by the Russian Science Foundation under grant 14-50-00005 and performed in Steklov Mathematical Institute of Russian Academy of Sciences. 


\section{References}

[1] V.V. Zharinov, Lie-Poisson structures over differential algebras, Theoret. and Math. Phys., 192:3 (2017), 1337-1349.

[2] P.J. Olver, Applications of Lie groups to differential equations, SpringerVerlag, New York, 1986, 1993.

[3] V.V. Zharinov, Lecture notes on geometrical aspects of partial differential equations, World Scientific Publishing Co. Inc., River Edge, NJ, 1992.

[4] N.H. Ibragimov, Transformation groups applied to mathematical physics, Reidel, Boston, 1985.

[5] V. V. Zharinov, Evolution systems with constraints in the form of zerodivergence conditions, Theoret. and Math. Phys., 163:1 (2010), 401-413.

[6] V. V. Zharinov, Bäclund transformations, Theoret. and Math. Phys., 189:3 (2016), 16811692.

[7] I.M. Gel'fand, I.Ya. Dorfman, Hamiltonian operators and algebraic structures related to them, Func. Anal. Appl., 13 (1979), 248-262.

[8] B.A. Dubrovin, S.P. Novikov, Hydrodynamics of weakly deformed soliton lattices. Differential geometry and Hamiltonian theory, Russian Math. Surveys, 44:6 (1989), 35-124.

[9] M. O. Katanaev, Killing vector fields and a homogeneous isotropic universe, Phys. Usp., 59:7 (2016), 689-700.

[10] G. A. Alekseev, Collision of strong gravitational and electromagnetic waves in the expanding universe, Phys. Rev. D, 93:6 (2016), 061501(R).

[11] A. P. Chugainova, V. A. Shargatov, Stability of discontinuity structures described by a generalized KdVBurgers equation, Comput. Math. Math. Phys., 56:2 (2016), 263-277.

[12] A. G. Kulikovskii, A. P. Chugainova, Study of discontinuities in solutions of the Prandtl-Reuss elastoplasticity equations, Comput. Math. Math. Phys., 56:4 (2016), 637-649. 
[13] V. A. Vassiliev, Local Petrovskii lacunas close to parabolic singular points of the wavefronts of strictly hyperbolic partial differential equations, Sb. Math., 207:10 (2016), 1363-1383.

[14] V. V. Kozlov, On the equations of the hydrodynamic type, J. Appl. Math. Mech., 80:3 (2016), 209-214.

[15] V. M. Buchstaber, Polynomial dynamical systems and the Korteweg-de Vries equation, Proc. Steklov Inst. Math., 294 (2016), 176-200.

[16] V. P. Pavlov, V. M. Sergeev, Fluid dynamics and thermodynamics as a unified field theory, Proc. Steklov Inst. Math., 294 (2016), 222-232.

[17] A. G. Kulikovskii, A. P. Chugainova, A self-similar wave problem in a Prandtl-Reuss elastoplastic medium, Proc. Steklov Inst. Math., 295 (2016), 179-189.

[18] A. T. Il'ichev, A. P. Chugainova, Spectral stability theory of heteroclinic solutions to the Korteweg-de Vries-Burgers equation with an arbitrary potential, Proc. Steklov Inst. Math., 295 (2016), 148-157.

[19] G. A. Alekseev, Integrable and non-integrable structures in EinsteinMaxwell equations with Abelian isometry group G2G2, Proc. Steklov Inst. Math., 295 (2016), 1-26. 